\title{
Partial stereotactic ablative boost radiotherapy in bulky non-small cell lung cancer: a retrospective study
}

Yun Bai'
Xian-shu Gao'
Shang-bin Qin'
Jia-yan Chen'
Meng-meng Su'
Qing Liu'
Xiu-bo Qin'2
Ming-wei Ma'
Bo Zhao'
Xiao-bin Gu'
Mu Xie'
Ming Cui'
Xin Qi'
Xiao-ying Li'
'Department of Radiation Oncology,
Peking University First Hospital,
Beijing, China; 'Department of Medical
Imaging, Peking University First
Hospital, Beijing, China

Correspondence: Xian-shu Gao Department of Radiation Oncology, Peking University First Hospital, No. 7 Xishiku Street, Xicheng District, Beijing 100034, China

Tel +86 I0 83575239

$\mathrm{Fax}+861066551788$

Email doctorgaoxs@126.com
This article was published in the following Dove Press journal: OncoTargets and Therapy

Purpose: Bulky non-small cell lung cancer (NSCLC) is difficult to achieve effective local control by conventionally fractionated radiotherapy (CRT). The present work aims to evaluate the safety and efficacy of partial stereotactic ablative boost radiotherapy (P-SABR) in bulky NSCLC.

Patients and methods: From December 2012 through August 2017, 30 patients with bulky NSCLC treated with P-SABR technique were analyzed. The P-SABR plan consisted of one partial SABR plan (5-9 Gy/f, 3-6 fractions) to gross tumor boost (GTVb), followed by one CRT plan to the planning target volume (PTV). GTVb was the max volume receiving SABR to guarantee the dose of organs-at-risks (OARs) falloff to about $3 \mathrm{~Gy} / \mathrm{f}$. The total dose of PTV margin was planned to above $60 \mathrm{~Gy}$. The simply CRT plans were created using the same planning parameters as the original plan, with the goal to achieve comparable OARs doses and PTV margin dose to the P-SABR plan. Dosimetric variables were acquired in both P-SABR and compared CRT plans. Toxicity, local control, and survival were also evaluated.

Results: Median follow-up in survivors was 10.3 months (range=2.3-39.4 months). Eleven patients (36.7\%) had partial response (PR) and ten patients $(33.3 \%)$ had stable disease (SD). Two-year overall survival was $55.6 \%$. Two-year local control rate was $85.7 \%$. No severe acute side effects $>$ CTCAE Grade III were observed. Compared to the simply CRT plan, P-SABR plans achieved similar doses to the OARs and Dmin, but increased dose at the isocenter, Dmean, Dmax, and biological equivalent dose (BED) significantly $(P<0.05)$. BED in the tumor center could reach 107.3 Gy (93.2-132 Gy). Patients with $\mathrm{B} 90 \geq 65 \%$ achieved a higher local control rate than those with $\mathrm{B} 90<65 \%(P=0.010)$.

Conclusion: This retrospective study suggests that P-SABR is feasible and well tolerated in bulky NSCLC. Local control rate is encouraging, especially for the B90 $\geq 65 \%$ group, which may due to the ability of P-SABR to optimize BED with equivalent toxicity.

Keywords: P-SABR, radiotherapy, NSCLC, bulky mass

\section{Introduction}

Lung cancer continues to be the leading cause of cancer-related mortality. Unfortunately, more than one-half of cases are diagnosed at a distant stage. ${ }^{1}$ The mass effect of a tumor in the thorax can cause symptoms such as cough, shortness of breath, chest pain, cardiac arrhythmia, esophageal obstruction, brachial plexopathy, superior vena cava compression, airway obstruction, postobstructive pneumonia, or even fatal hemoptysis.

As is known to all, it is generally challenging to achieve effective local control in bulky lung cancer. ${ }^{2,3}$ Mounting evidence now suggests that stereotactic ablative radiotherapy (SABR), also known as stereotactic body radiotherapy (SBRT), which involves 
the delivery of very high individual doses of radiation to tumors in various extra-cranial sites with high precision, is capable to achieve local control compared to surgery occasionally. Unfortunately, SABR can only be safely administrated in small cancer confined to appropriate early stage non-small cell lung cancer (NSCLC) and oligometastases. ${ }^{4,5}$ For advanced NSCLC, SBRT alone increases radiation toxicity significantly, thus is not recommended. ${ }^{6-8}$

Partial stereotactic ablative boost radiotherapy (P-SABR), which combines conventionally fractionated radiotherapy (CRT) with partial SABR boost, can deliver high cumulative doses of radiation to bulky tumor, while overcoming the limitation of normal tissue tolerance. This study analyzed the results of treatments in bulky NSCLC receiving P-SABR.

\section{Patients and methods}

\section{Patients selection}

Eligible patients had a lesion with a diameter longer than $4.5 \mathrm{~cm}$, based on computed tomography (CT). Eligible patients had to undergo and complete P-SABR as well as concurrent chemotherapy. Additionally, patients refused or who were intolerable to undergo concurrent chemotherapy were also eligible. Between December 2012 and August 2017, a total of 30 patients fulfilled the above criteria and were analyzed in this retrospective single institution study. Tumor characteristics, patient history, clinical course, response, and toxicities were extracted from the database and the patient's medical records. The study was approved by the Peking University First Hospital National Unit of Clinical Trial Ethics Committees (Protocol number 2017/57). No patient consent was required since this was a retrospective study and the data collection was standard care. All medical data were de-identified. All presentations of case reports have consent for publication.

\section{P-SBRT technique}

Patients were immobilized with the thermoplastic film. Respiratory-acquired 4-dimensional (4D) CT simulation was performed to define tumor motion. The gross tumor volume (GTV), comprising the known extent of disease (primary and nodal) on imaging (CT/PET-CT) and pathologic assessment, was delineated in the CT pulmonary window. The clinical target volume (CTV) includes regions of presumed microscopic extent or dissemination. An internal target volume (ITV) was the sum of the GTV/CTV positions in inhalation and exhalation. The planning target volume (PTV) consisted of the CTV with a $5 \mathrm{~mm}$ margin expansion. The gross tumor boost volume (GTVb) was the maximum volume receiving SABR, while ensuring the organs-at-risk (OAR) dose falloff to about $3 \mathrm{~Gy} / \mathrm{f}$ (Figure 1). Using intensity modulated radiotherapy (IMRT or volumetric modulated arc therapy, VMAT), plans were generated so that $\geq 95 \%$ of the PTV was encompassed by $\geq 100 \%$ of the prescription dose.

Normal tissue dose constraints (lung, heart, esophagus, and spinal cord) for partial SABR boost plans and composite plans of partial SBRT boost were based on commonly used constraints. The plans were generated by the following treatment planning programs: XiO (V5.0, Elekta, Stockholm, Sweden), Monaco (V3.2, Elekta), Monaco (V5.0, Elekta), Monaco (V5.11, Elekta), Eclipse (V13.5, Varian Medical Systems, Palo Alto, CA). Doses were calculated using Superposition, Monte Carlo, and Analytical Anisotropic Algorithm (AAA) dose calculation algorithms, respectively, in XIO, Monaco, and Eclipse systems. All patients were treated five times per week with $6 \mathrm{MeV}$ photons. Cone-beam CT was used for daily image-guidance to verify the position of the target in all patients before treatment.

The P-SABR plan consisted of two plans, the SABR component delivers the GTVb dose of 15-48 Gy in 3-6 fractions, followed by the CRT component delivering the PTV dose of 38-60 Gy in 15-33 fractions. The dose of GTVb was planned to about 76.1 Gy (69-89.2 Gy). The total dose of PTV margin was planned to about 64 Gy (56-72 Gy) (Table 1). The mean biological equivalent dose (BED) at the isocenter was 107.3 Gy (93.2-132 Gy).
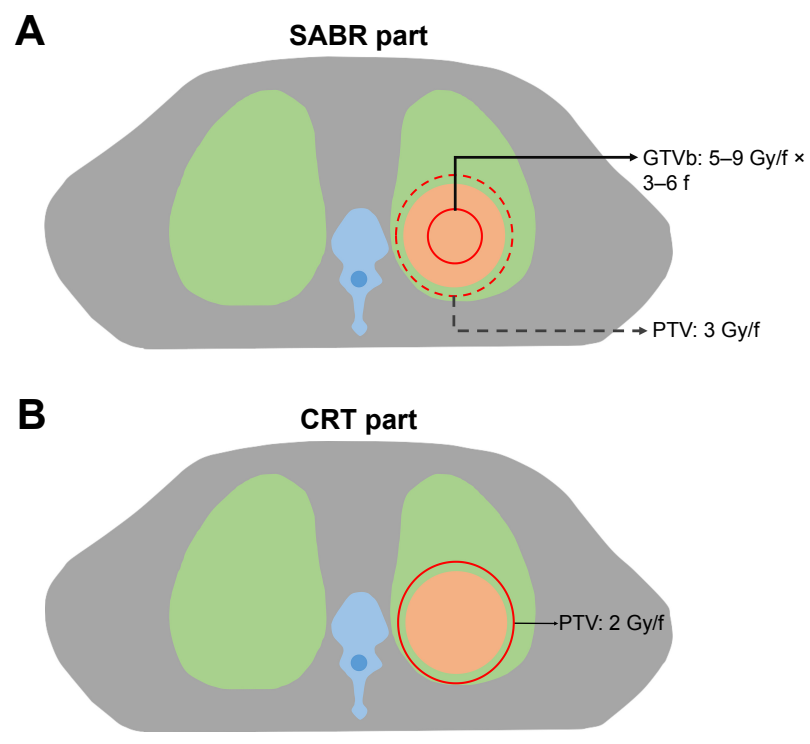

Figure I Schematic diagram of the P-SABR. The P-SABR plan combines a partial SABR boost plan and a CRT plan. (A) The SABR (5-9 Gy per fraction) was delivered to the max tumor volume, while the OAR dose fell off to $3 \mathrm{~Gy} / \mathrm{f}$. (B) The CRT component was delivered afterwards, ensuring the total PTV margin dose to about 64 Gy.

Abbreviations: P-SABR, Partial Stereotactic Ablative Boost Radiotherapy; SABR, Stereotactic Ablative Radiotherapy; CRT, Conventionally Fractionated Radiotherapy; OAR, Organs-At-Risk; PTV, Planning Target Volume. 
Table I Treatment schemes of P-SABR

\begin{tabular}{ll}
\hline Parameters & Median (range) \\
\hline SABR plans & \\
Single dose (Gy/fraction) & $6(5-9)$ \\
Fractions & $4(3-6)$ \\
Total dose (Gy) & $24(15-48)$ \\
CRT plans & \\
Single dose (Gy/fraction) & $2(1.8-3)$ \\
Fractions & $26(15-33)$ \\
Total dose (Gy) & $52(38-60)$ \\
Dose & \\
GTVb (Gy) & $76.1(69-89.2)$ \\
Total dose in the margin of PTV (Gy) & $64(56-72)$ \\
\hline
\end{tabular}

Abbreviations: P-SABR, Partial Stereotactic Ablative Boost Radiotherapy; SABR, Stereotactic Ablative Radiotherapy; CRT, Conventionally Fractionated Radiotherapy; GTVb, Gross Tumor boost; PTV, Planning Target Volume.

\section{Comparative CRT plan and dosimetric variables}

All cases were redesigned using the same planning objectives. The simply CRT plans were designed to generate the same dose and fractions of PTV as the comparative P-SABR plan. The objective of the simply CRT plans was to achieve comparable OAR doses to those of the original P-SABR plan. The simply CRT plans were generated by the treatment planning program, the same as the original, and verified or improved by a medical physicist.

Dose-volume histograms (DVHs) were compared between P-SABR and CRT plans for the following dosimetric metrics. Dm means the maximum dose received by $\mathrm{m} \%$ volume. For example: D02 means the maximum dose received by $2 \%$ volume. $\mathrm{Vn}$ means the percentage of the structure receiving doses $>$ n. For example: V65 means the volume of the structure receiving doses $>65 \mathrm{~Gy}$. Conformity index (CI) was calculated by SALT (SaintAnne, Lariboisière, Tenon) and van't Riet. ${ }^{9}$ A CI equal to 1 corresponds to ideal conformation. A CI $>1$ indicates that the irradiated volume is greater than the target volume and includes healthy tissues. If the $\mathrm{CI}$ is $<1$, the target volume is only partially irradiated. The higher the Homogeneity index (HI), the plan is less homogeneous. $\mathrm{BED}$ was calculated using the linear-quadratic equation. Bi means the volume of the structure receiving doses $>i$. For example: B80 means the volume of the structure receiving doses $>80$ Gy.

$$
\mathrm{CI}_{\mathrm{SALT}}=\mathrm{TVRI} / \mathrm{TV}
$$

where TVRI $=$ Target volume covered by the reference isodose; and TV = Target volume.

$$
\mathrm{CN}=(\mathrm{TVRI} / \mathrm{TV}) \times(\mathrm{TVRI} / \mathrm{VRI})
$$

where $\mathrm{CN}=$ Conformation number proposed by van't Riet; and VRI $=$ Volume of the reference isodose.

$$
\begin{aligned}
& \mathrm{HI}=[(\mathrm{D} 02-\mathrm{D} 98) / \mathrm{D} 50] \\
& \mathrm{BED}=\mathrm{Nd} \times(1+\mathrm{d} / \alpha / \beta)
\end{aligned}
$$

where $\mathrm{N}=$ fractionation number, $\mathrm{d}=$ daily dose, and $\alpha / \beta=10$ for NSCLC.

\section{Follow-up}

Patients were routinely followed up at 1 month, 3 months, 6 months, 9 months, 1 year, and about twice annually thereafter. Due to the palliative setting, follow-up was often based on a limited and clinically necessary follow-up, and often no visit to the hospital was possible. Toxicities, including acute toxicity within 3 months and late toxicity beyond 3 months of P-SABR, were assessed with the Common Terminology Criteria for Adverse Events (CTCAE) version 4.03. Follow-up CT scans were performed at each visit. Radiological response was determined by radiologists according to standard Response Evaluation Criteria In Solid Tumors (RECIST) criteria version 1.1, defining a complete response (CR) as a disappearance of all target lesions, and a reduction in the short axis to $<10 \mathrm{~mm}$ in any pathological lymph nodes. A partial response (PR) was defined as at least a $30 \%$ decrease in the sum of diameters of target lesions, taking as a reference the baseline sum diameters. Progressive disease (PD) is scored with a minimum of $20 \%$ increase in the sum of diameters of target lesions, and stable disease (SD), showing neither sufficient shrinkage to qualify for PR nor sufficient increase to qualify for PD. Local control rate was determined according to the decrease of the target lesion treated by P-SABR. Defining a CR as a disappearance of the target lesion, a PR was defined as at least a $30 \%$ decrease in the diameter of target lesion, taking as reference the baseline sum diameter. PD is scored with a minimum of $20 \%$ increase in the diameter of target lesions, and SD showed neither sufficient shrinkage to qualify for PR nor a sufficient increase to qualify for PD. Defining CR and PR as a high local control rate, local response was defined as a tumor treated by P-SABR shrinkage and no tumor progress during follow-up.

\section{Statistical analysis}

We used statistical software SPSS 23.0 for the analyses. Summary statistics were provided with frequency count 
and percentage for categorical variables, and mean, SD, median, and range for continuous variables. We used Paired $t$-test to estimate the dosimetric results between compared radiotherapy plan. We calculated overall survival from the date of treatment to the last contact date (death date or last follow-up date, at which point patients who were still alive were censored). We calculated time to local recurrence from the date of treatment to the date of first recurrence. Patients who did not have recurrence or metastases were censored at the date of death or last follow-up. We used the Kaplan-Meier method to estimate overall survival and time to local recurrence, and used log-rank tests to evaluate differences in time-to-event outcomes with two-sided $P$-values. We classified $P$-values $<0.05$ as statistically significant.

\section{Results}

\section{Patient characteristics}

Detailed patients' characteristics are summarized in Table 2. Twenty-seven patients (90\%) were male, three patients (10\%)

Table 2 Patients and treatment characteristics

\begin{tabular}{ll}
\hline Characteristic & Total, $\mathbf{n}(\%)$ \\
\hline Patient characteristics ( $\mathrm{n}=30$ patients) & \\
Age (years), median (range) & $64.5(40-85)$ \\
Gender & \\
Male & $27(90)$ \\
Female & $3(10)$ \\
Staging & \\
IIB & $3(10)$ \\
IIIA & $9(30)$ \\
IIIB & $6(20)$ \\
IV & $12(40)$ \\
T stage & \\
T2 & $3(10)$ \\
T3 & $14(46.7)$ \\
T4 & $13(43.3)$ \\
Target location & \\
Right Upper & $9(30)$ \\
Right Lower & $4(13.3)$ \\
Left Upper & $1 \mathrm{I}(36.7)$ \\
Left Lower & $6(20)$ \\
Concurrent treatment & \\
Non & $14(46.7)$ \\
Chemotherapy & $16(53.3)$ \\
Histology & \\
Squamous cell carcinoma & $17(56.7)$ \\
Adenocarcinoma & $5(16.7)$ \\
Large cell carcinoma & $1(3.3)$ \\
Neuroendocrine carcinoma & $1(3.3)$ \\
Others & $2(6.7)$ \\
Unknown & $4(13.3)$ \\
Tumor volume (cm $),$ median (range) & $138.07(35.55-495.50)$ \\
Tumor size (cm), median (range) & $8.08(4.62-14.64)$ \\
Follow-up (months), median (range) & $10.3(2.3-39.4)$ \\
\hline
\end{tabular}

was female. Median age at treatment was 64.5 years (40-85 years). Twenty-six patients were pathologically diagnosed as having NSCLC, including 17 patients $(56.7 \%)$ with squamous cell carcinoma, five patients (16.7\%) with adenocarcinoma, one (3.3\%) with large cell carcinoma, one $(3.3 \%)$ with neuroendocrine carcinoma, and two patients $(6.7 \%)$ were other types. Another four patients, failed to obtain pathological biopsy, were clinically diagnosed as having NSCLC, based on clinical information such as elevated tumor marker levels, an increase in the maximum standardized uptake value on ${ }^{18} \mathrm{~F}$-FDG PET/CT, and successive enlargements on CT images. Three patients (10\%) had stage IIB(T3N0M0), nine patients (30\%) had stage IIIA, six patients $(30 \%)$ had stage IIIB, and 12 patients $(40 \%)$ had stage IV according to AJCC Cancer Staging Manual, Seventh Edition (2010). Median volume of the tumor treated by P-SABR was $138.07 \mathrm{~cm}^{3}\left(35.55-495.50 \mathrm{~cm}^{3}\right)$, with a median maximal diameter of $8.08 \mathrm{~cm}(4.62-14.64 \mathrm{~cm})$.

\section{Response and survival outcomes}

Median follow-up in survivors was 10.3 months (range= 2.3-39.4 months) from the beginning of RT. Nineteen patients died, five patients were censored, and six patients were still alive at the last follow-up date. One-year overall survival was $88.2 \%$. Two-year overall survival was $55.6 \%$ (Figure 2A). Local response was achieved in 27 of these 30 patients (90\%). Eleven patients (36.7\%) had PR, 10 patients $(33.3 \%)$ had SD, and nine patients $(30 \%)$ had PD according to the RECIST 1.1 criteria. Seven patients $(23.3 \%)$ had local response but systemic progression, and three patients $(10 \%)$ presented with a RT-in-fieldprogression. An example of patient response is shown in Figure 3. One-year local control rate was 100\%. Two-year local control rate was $85.7 \%$. (Figure 2B). Patients with B $90 \geq 65 \%$ achieved a higher local control rate than those with $\mathrm{B} 90<65 \%$ (median survival=15.2 months vs 3.5 months, $\mathrm{CI}=6.8-23.6$ and 2.2-4.9 months, respectively; $P=0.010$; Figure 2C). Patients with $\mathrm{B} 80 \geq 90 \%$ achieved a higher local control rate than those with $\mathrm{B} 80<90 \%$ (median survival=14.9 months vs 3.5 months, $C I=3.4-26.3$ and 1.8-5.2 months, respectively; $P=0.045$; Figure 2D).

\section{Tolerability and side effects}

Therapy was completed as scheduled in all the cases, thus an acceptable tolerability of the RT regimen can be seen. No severe acute side effects $>$ CTCAE Grade III were observed. Grade 3 acute myelosuppression was present in one patient 

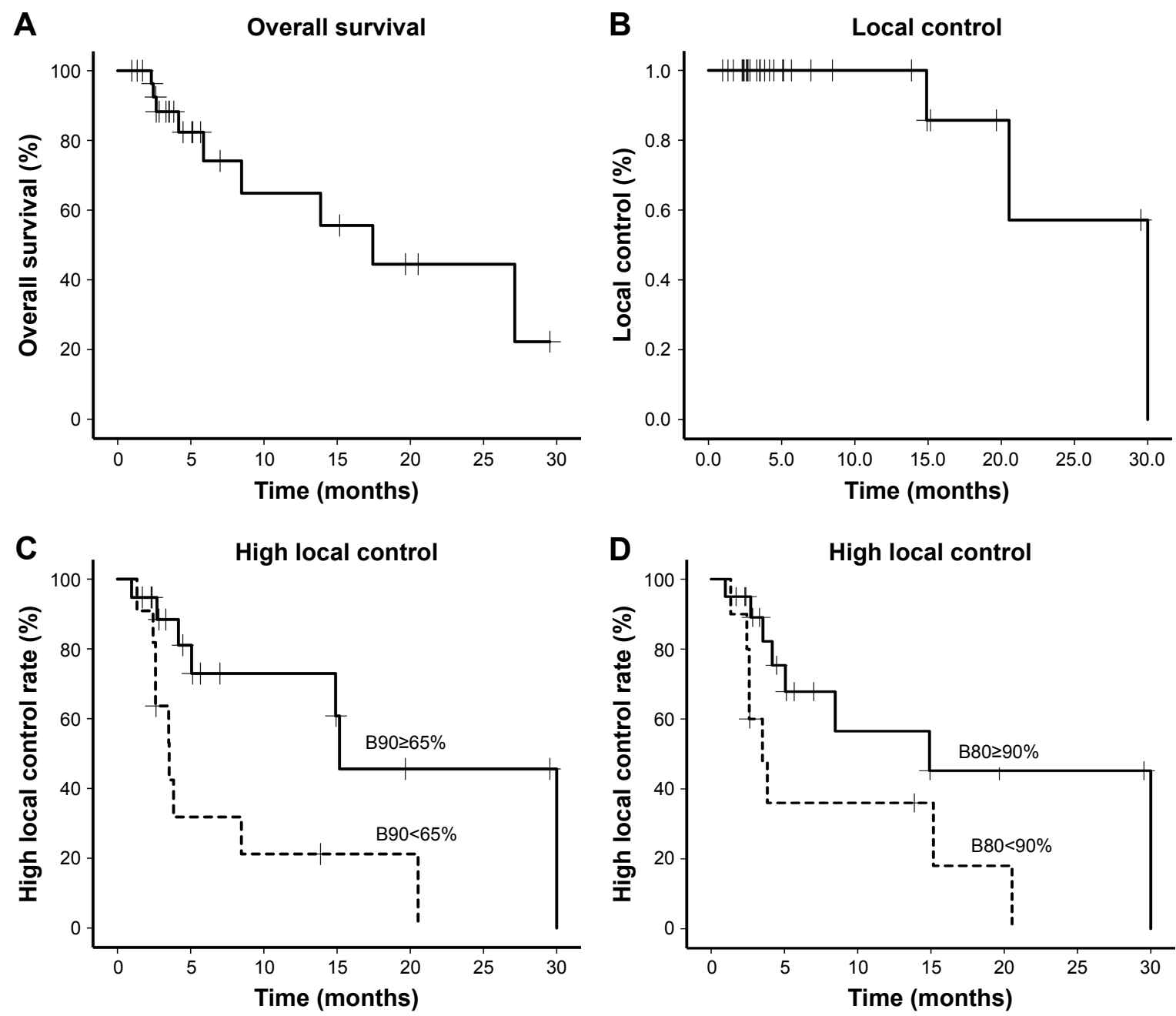

Figure 2 (A) Kaplan-Meier plots of overall survival for all patients. One-year overall survival was $88.2 \%$. Two-year overall survival was $55.6 \%$. (B) Kaplan-Meier plots of local control for all patients. One-year local control rate was 100\%. Two-year local control rate was $85.7 \%$. (C) Patients with B90 $\geq 65 \%(\mathrm{n}=19)$ achieved a higher local control rate than those with $B 90<65 \%(n=1)$ (median survival=15.2 months vs 3.5 months, $\mathrm{Cl}=6.8-23.6$ and $2.2-4.9$ months, respectively; $P=0.010$ ). (D) Patients with $B 80 \geq 90 \%$ ( $=20$ ) achieved a higher local control rate than those with $\mathrm{B} 80<90 \%(\mathrm{n}=10)$ (median survival=14.9 months vs 3.5 months, $\mathrm{Cl}=3.4-26.3$ and $\mathrm{I} .8-5.2$ months, respectively; $P=0.045$ ).

treated by P-SABR concurrent with cisplatin. Two patients, whose V5 were $61.41 \%$ and $69.89 \%$ and V20 were $19.88 \%$ and $29.80 \%$, respectively, developed Grade 3 acute pneumonitis treated by P-SABR alone. Long-term follow-up, Grade 4 late esophagitis, tracheoesophageal fistula, was present in one patient whose tumor had invaded the esophagus before RT. One patient treated by P-SABR after four cycles of Gemcitabine and cisplatin, whose V5 was $62.07 \%$ and V20 was $23.67 \%$, developed Grade 3 late pneumonitis. The other 12 patients didn't complain of any $\geq$ Grade II long-term side-effects (Table 3).

\section{Dosimetric advantages}

Tables 4 and 5 summarize dosimetric results for two plans of the 30 patients. P-SABR had a significantly higher HI index $(P<0.001)$, with a similar CI index $(P>0.5)$.
No difference was found in most normal tissue doses between P-SABR and the simply CRT plans $(P>0.1)$, except V5 of the lungs (47.548\% for P-SABR vs $44.552 \%$ for CRT, $P=0.021)$. The dose of the contralateral lung was slightly higher in the P-SABR plan. However, V20 and mean dose of lungs were not different significantly $(P>0.1)$. No differences were found in Dmin and D98 of PTV between P-SABR and the simply CRT plans $(P>0.1)$. Compared to the CRT plan, P-SABR plans increased dose at the isocenter, BED at the isocenter, Dmean, Dmax, D50, and $\mathrm{D} 02$ by $17.89 \%, 26.31 \%, 7.75 \%, 14.39 \%, 7.81 \%$, and $14.79 \%$, respectively $(P<0.001)$ (Figure 4$)$. Similarly, B80, B90, B100, B110, B120, V65, V70, V75, and V80 were improved by $27.91 \%, 74.11 \%, 96.10 \%, 99.90 \%$, $100.00 \%, 33.72 \%, 61.64 \%, 68.54 \%$, and $80.51 \%$, respectively $(P<0.05)$. 
A

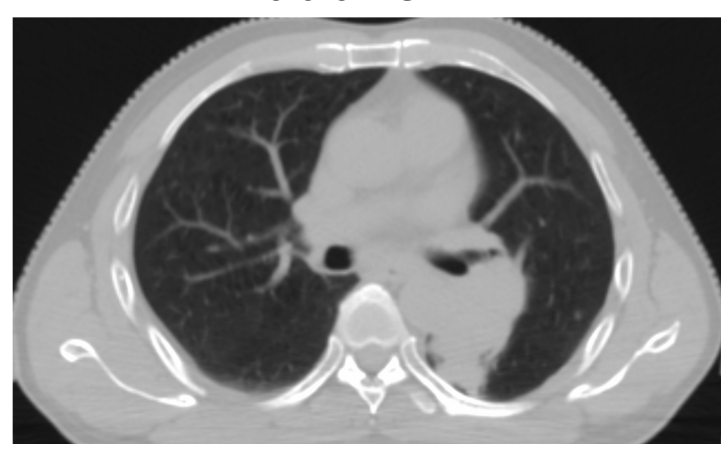

C

7 months after P-SABR

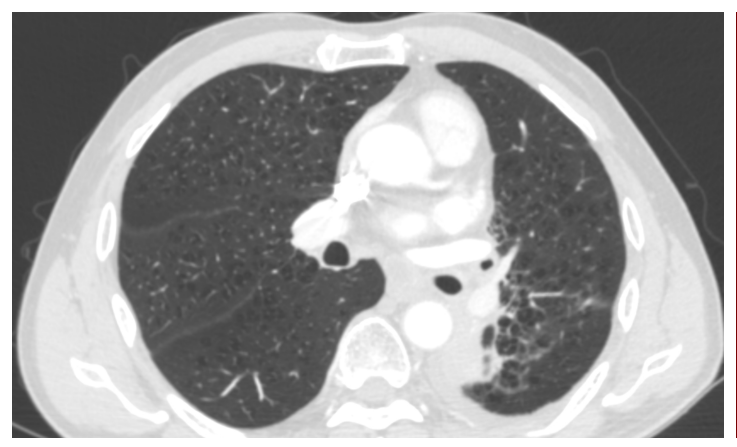

B

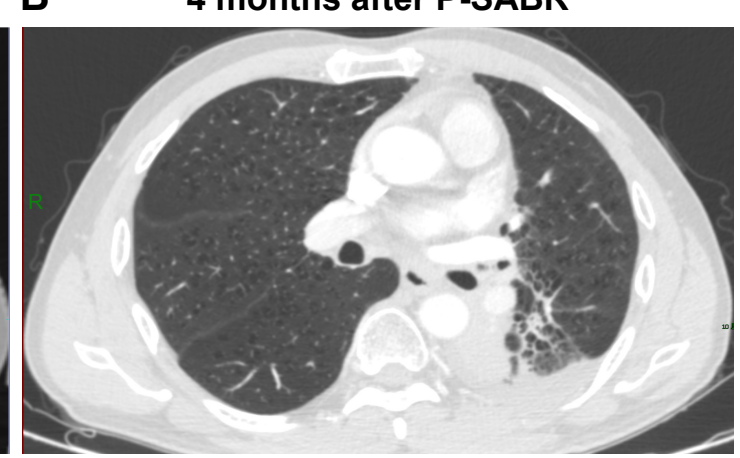

D 18 months after P-SABR

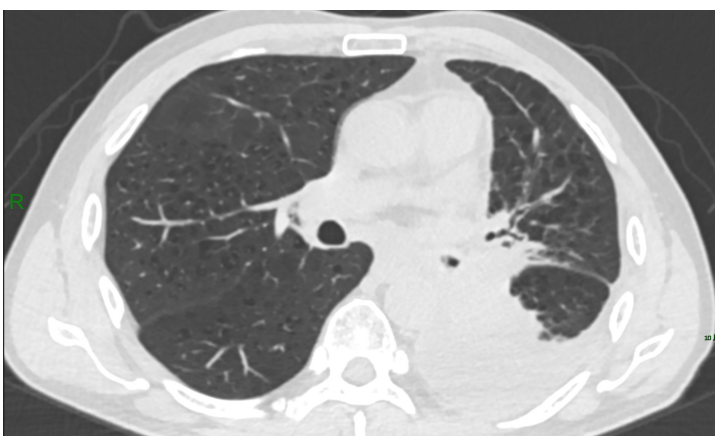

Figure 3 A 56-year-old male with squamous cell cancer of the left lung (T3N2M0). Before (A) and 4 months (B), 7 months (C), and I8 months (D) after P-SABR (SABR plans: $6 \mathrm{~Gy} / \mathrm{f} \times 4 \mathrm{f}$, CRT plans: $2.2 \mathrm{~Gy} / \mathrm{f} \times 26 \mathrm{f} \rightarrow$ Dose: GTVb 8I.2 Gy/PTV $69.2 \mathrm{~Gy} / 30 \mathrm{f}$ ).

Abbreviations: P-SABR, Partial Stereotactic Ablative Boost Radiotherapy; SABR, Stereotactic Ablative Radiotherapy; CRT, Conventionally Fractionated Radiotherapy; GTVb, Gross Tumor boost; PTV, Planning Target Volume.

\section{Discussion}

Therapeutic effects of CRT in locally advanced lung cancer are unsatisfactory, the overall survival is $15 \%-30 \%$, and the local recurrence rate is $30 \%-55 \%$ at 5 years. ${ }^{10,11}$ As the tumor grows larger, the overall survival and local control rate decrease. Despite introduction of chemotherapy agents, survival remains poor. ${ }^{12,13}$ Nowadays, SABR can deliver a highly conformal radiation dose in only a few treatments, resulting in a biologically more potent dose of radiation without a significant prolongation of treatment time..$^{14,15}$

Table 3 Toxicity* after P-SABR for NSCLC patients

\begin{tabular}{lllll}
\hline Parameters & $\begin{array}{l}\text { Grade II } \\
\text { n (\%) }\end{array}$ & $\begin{array}{l}\text { Grade III } \\
\text { n (\%) }\end{array}$ & $\begin{array}{l}\text { Grade IV } \\
\text { n (\%) }\end{array}$ & $\begin{array}{l}\text { Grade V } \\
\text { n (\%) }\end{array}$ \\
\hline Acute toxicity & & & & \\
$\quad \begin{array}{llll}\text { Myelosuppression } \\
\text { Pneumonitis }\end{array}$ & $\mathrm{I}(3.3)$ & $\mathrm{I}(3.3)$ & - & - \\
$\quad 5(16.7)$ & $2(6.7)$ & - & - \\
Esophagitis & $4(13.3)$ & - & - & - \\
Late toxicity & & & & - \\
$\quad \begin{array}{l}\text { Pneumonitis } \\
\text { Esophagitis }\end{array}$ & - & $\mathrm{I}(3.3)$ & - & - \\
\hline
\end{tabular}

Note: *Toxicities, including acute toxicity within 3 months and late toxicity beyond 3 months of P-SABR, were assessed with the CTCAE version 4.03.

Abbreviations: P-SABR, Partial Stereotactic Ablative Boost Radiotherapy; NSCLC, non-small cell lung cancer; CTCAE, Common Terminology Criteria for Adverse Events.
Mounting evidence demonstrates that SABR could increase the antitumor immune effect. ${ }^{16-18}$ In addition, the biological effects of SABR on vasculature may further improve tumor control. ${ }^{19,20}$ SABR is proved to be capable of achieving high local control equivalent to surgery in T1 or T2N0M0 NSCLC. ${ }^{21,22}$ However, increased toxicities reduce the application of SABR in bulky lung cancer. ${ }^{6-8}$ Thus, novel approaches are urgently needed to effectively control the disease.

Salazar et $\mathrm{a}^{23}$ published a similar retrospective study in regard of once-weekly SBRT in advanced lung cancer patients. SBRT was used as a boost to the primary tumor with three fractions of 7.5 Gy once-weekly at the PTV (10.7 Gy/ fraction at the isocenter) after daily conventionally fractionated RT (45 Gy/25 f). The overall BED of this combination was $119.6 \mathrm{~Gy}_{10}$ at the isocenter. The median follow-up was 38 months. No Grade 3-4 acute toxicities developed in any patient. Late toxicity, all Grade 2, developed in 3\% of patients. This study supports the hypothesis that SBRT boost is well tolerated in advanced lung cancer. In theory, tumors treated by once-weekly SABR shrink more significantly than by daily SABR, thus a higher tumor volume would be included in daily SABR, which might increase the local control rate of RT consequently. Therefore, daily SABR was applied in our article and the 2-year local control rate was as 
Table 4 Comparison of OAR doses between P-SABR and CRT plans

\begin{tabular}{|c|c|c|c|c|c|}
\hline \multirow[t]{2}{*}{ Parameters } & \multicolumn{2}{|l|}{ P-SABR } & \multicolumn{2}{|l|}{ CRT } & \multirow[t]{2}{*}{$P$-value } \\
\hline & Mean & SD & Mean & SD & \\
\hline \multicolumn{6}{|l|}{ Lung } \\
\hline Double lungs Dmean (cGy) & $\mathrm{I}, 064.070$ & 492.197 & $\mathrm{I}, 048.5 \mathrm{I} \mathrm{I}$ & 444.336 & 0.834 \\
\hline Double lungs V5 (\%) & 47.548 & 21.606 & 44.552 & 21.530 & 0.021 \\
\hline Ipsilateral lung V5 (\%) & 56.962 & 22.304 & 56.906 & 22.256 & 0.923 \\
\hline Contralateral lung V5 (\%) & 41.718 & 23.032 & 39.129 & 24.495 & 0.011 \\
\hline Double lungs V20 (\%) & 17.833 & 8.217 & 16.703 & 8.649 & 0.311 \\
\hline \multicolumn{6}{|l|}{ Spinal cord } \\
\hline$D \max (c G y)$ & $3,445.822$ & $\mathrm{I}, 0 \mathrm{I} 5.766$ & $3,577.391$ & 784.482 & 0.337 \\
\hline D02 (cGy) & $3,130.748$ & 773.972 & $3,265.096$ & 800.136 & 0.140 \\
\hline \multicolumn{6}{|l|}{ Heart } \\
\hline Dmean (cGy) & $1,046.956$ & 938.947 & $\mathrm{I}, 053.772$ & $1,010.143$ & 0.894 \\
\hline V30 (\%) & $\mathrm{II} .797$ & 13.404 & 12.186 & 14.219 & 0.297 \\
\hline V40 (\%) & 5.334 & 6.490 & 5.668 & 7.300 & 0.394 \\
\hline V45 (\%) & 3.514 & 4.665 & 3.540 & 4.774 & 0.890 \\
\hline V60 (\%) & 1.038 & 2.143 & $0.86 I$ & 1.903 & 0.510 \\
\hline \multicolumn{6}{|l|}{ Esophagus } \\
\hline Dmean (cGy) & $2,484.667$ & I,3।8.29| & $2,422.033$ & $\mathrm{I}, 602.254$ & 0.810 \\
\hline Dmax (cGy) & $6,241.871$ & $1,615.026$ & $5,789.262$ & $|, 8| 4.050$ & 0.142 \\
\hline V45 (\%) & 25.120 & 23.288 & 25.420 & 24.097 & 0.788 \\
\hline
\end{tabular}

Notes: $\mathrm{Dm}$ means the maximum dose received by $\mathrm{m} \%$ volume. For example, D02 means the maximum dose received by $2 \%$ volume. $\mathrm{V} n$ means the percentage of the structure receiving doses $>\mathrm{n}$. For example, V5 means the volume of the structure receiving doses $>5 \mathrm{~Gy}$.

Abbreviations: OAR, Organs-At-Risk; P-SABR, Partial Stereotactic Ablative Boost Radiotherapy; CRT, Conventionally Fractionated Radiotherapy; Dmean, Mean dose; Dmax, Maximize dose.

Table 5 Comparison of dosimetric metrics between P-SABR and CRT plans

\begin{tabular}{|c|c|c|c|c|c|}
\hline \multirow[t]{2}{*}{ Parameters } & \multicolumn{2}{|c|}{ P-SABR } & \multicolumn{2}{|l|}{ CRT } & \multirow[t]{2}{*}{$P$-value } \\
\hline & Mean & SD & Mean & SD & \\
\hline Dmin (Gy) & 50.597 & 8.615 & 51.459 & 7.562 & 0.331 \\
\hline D98 (Gy) & 58.485 & 12.484 & 61.616 & 4.862 & 0.186 \\
\hline Dmean (Gy) & 72.735 & 6.603 & 67.097 & 4.682 & 0.000 \\
\hline D50 (Gy) & 72.853 & 7.562 & 67.166 & 5.100 & 0.000 \\
\hline Dmax (Gy) & 85.931 & 7.236 & 73.564 & 6.000 & 0.000 \\
\hline D02 (Gy) & 83.751 & 7.251 & 71.361 & 5.496 & 0.000 \\
\hline $\begin{array}{l}\text { Dose (at the } \\
\text { isocenter) (Gy) }\end{array}$ & 78.450 & 5.196 & 64.413 & 4.634 & 0.000 \\
\hline V65 (\%) & 86.514 & 17.768 & 57.340 & 38.404 & 0.001 \\
\hline V70 (\%) & 67.636 & 22.781 & 25.947 & 40.378 & 0.000 \\
\hline V75 (\%) & 46.352 & 27.238 & 14.584 & 30.187 & 0.000 \\
\hline V80 (\%) & 24.649 & 29.683 & 4.805 & 17.869 & 0.005 \\
\hline $\begin{array}{l}\text { BED (at the } \\
\text { isocenter) (Gy) }\end{array}$ & 107.339 & 9.826 & 79.099 & 5.733 & 0.000 \\
\hline B80 (\%) & 87.147 & 20.994 & 62.828 & 32.624 & 0.000 \\
\hline B90 (\%) & 64.339 & 26.875 & 16.660 & 29.451 & 0.000 \\
\hline BIO0 (\%) & 41.549 & $30.34 I$ & 1.620 & 4.835 & 0.000 \\
\hline BIIO (\%) & 19.237 & 27.460 & 0.019 & 0.098 & 0.001 \\
\hline BI 20 (\%) & 7.065 & 15.516 & 0.000 & 0.000 & 0.026 \\
\hline $\mathrm{Cl}(\mathrm{SALT})$ & 0.880 & 0.153 & 0.859 & 0.248 & 0.708 \\
\hline $\mathrm{Cl}$ (Van) & 0.688 & 0.278 & 0.678 & 0.283 & 0.873 \\
\hline $\mathrm{HI}$ & 0.353 & 0.191 & 0.146 & 0.090 & 0.000 \\
\hline
\end{tabular}

Notes: Dm means the maximum dose received by $\mathrm{m} \%$ volume. For example, D02 means the maximum dose received by $2 \%$ volume. $\mathrm{Vn}$ means the percentage of the structure receiving doses $>$ n. For example, $V 65$ means the volume of the structure receiving doses $>65 \mathrm{~Gy}$. Bi means the volume of the structure receiving doses $>i$. For example, B80 means the volume of the structure receiving doses $>80 \mathrm{~Gy}$. Abbreviations: P-SABR, Partial Stereotactic Ablative Boost Radiotherapy; CRT, Conventionally Fractionated Radiotherapy; Dmin, Minimum dose; Dmean, Mean dose Dmax, Maximum dose; BED, Biologically effective dose; $\mathrm{Cl}$ (SALT), Conformity index calculated by SALT (Saint-Anne, Lariboisière, Tenon); Cl (Van), Conformity index calculated by van't Riet; HI, Homogeneity index. high as $85.7 \%$, even stage IV NSCLC were $40 \%$. The local control rate for stage II, IIIA, IIIB, and IV patients was $67 \%$, $73 \%, 47 \%$, and $0 \%$ in once-weekly SABR, respectively.

A similar published technique was the SABR boost after concurrent chemoradiation. Feddock et a ${ }^{24}$ reported a phase 2 study reporting 35 locally advanced NSCLC patients who were treated by standard fractionated chemoradiation to a dose of $60 \mathrm{~Gy}$, following by SBRT boost ( $20 \mathrm{~Gy} / 2 \mathrm{f}$ ) to the primary tumor only. At a median follow-up time of 13 months, local control at the primary tumor site was $83 \%$. Additional retrospective data came from Karam et al, ${ }^{25}$ including 16 patients treated with chemoradiation to 45-60 Gy who underwent a SABR boost (20-30 Gy/5 f) to residual disease on PET imaging at 6 weeks. With a median follow-up time of 14 months, local control was $78 \%$. These studies demonstrated that a SABR boost to the residual tumor $(<5 \mathrm{~cm})$ can improve disease control which was similar to ours. Theoretically, a partial SABR boost before CRT might decrease OARs doses more than a SABR boost after CRT, which would decrease side-effects sequentially. In our research, one Grade 3 late pneumonitis and one Grade 4 late esophagitis, whose tumor had invaded the esophagus before RT were reported. Feddock et $\mathrm{al}^{24}$ reported one patient with grade 3 late pneumonitis, two patients with grade 2 late pneumonitis, and two patients experienced cavitary recurrence and fatal hemoptysis. Karam et $a^{25}$ reported one patient with nonfatal hemoptysis.

Our results show that P-SABR increases the dose and BED with equivalent toxicity in bulky NSCLC. Specifically, 
A

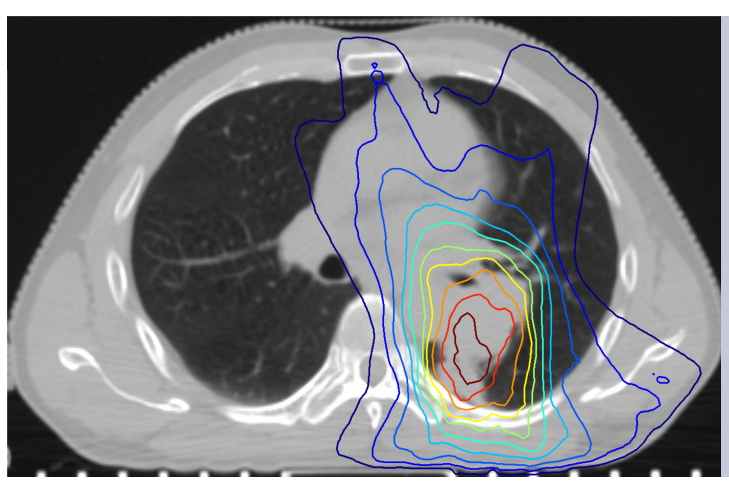

C 100

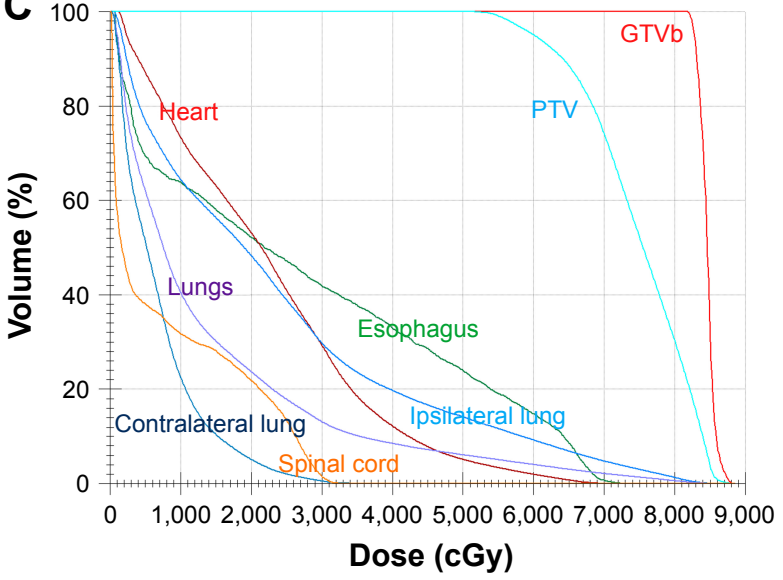

B

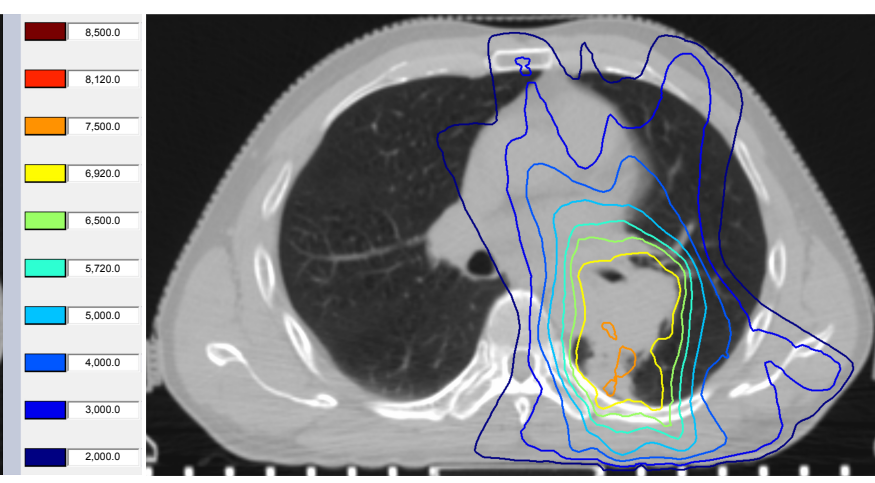

D 100

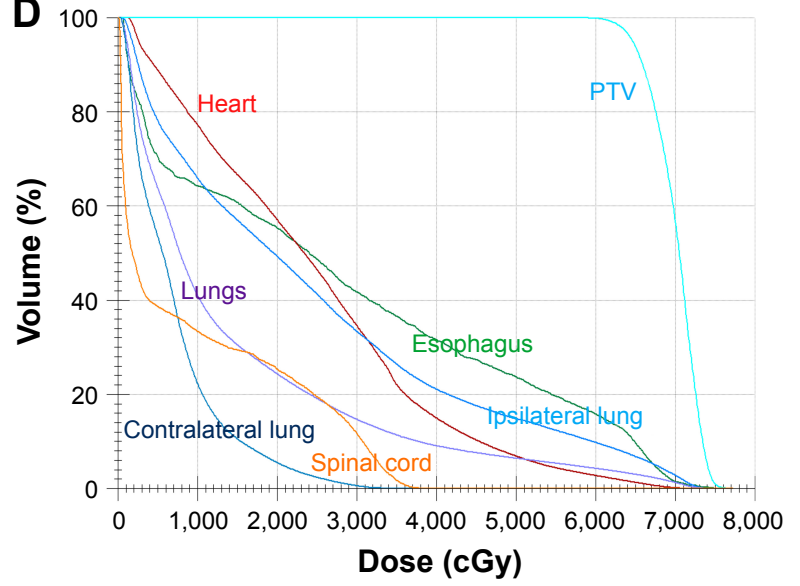

Figure 4 The dose distribution curves of a P-SABR plan (A) and comparative CRT plan (B). The isodose lines, from outer to inner, represent 20, 30, 40, 50, 57.2, 60, 69.2, 75, 8I.2, and 85 Gy, respectively. The DVH of this P-SABR plan (C) and comparative CRT plan (D). Detailed dosimetric results including P-values are described in Tables 4 and 5 .

Abbreviations: P-SABR, Partial Stereotactic Ablative Boost Radiotherapy; CRT, Conventionally Fractionated Radiotherapy; DVH, Dose Volume Histogram.

P-SABR can deliver higher radiation doses to the bulky tumor center, including Dmean, Dmax, B80, B90, B100, B110, B120, V65, V70, V75, and V80 while it doesn't increase Dmin and the surrounding normal tissue dose. As a result, no severe side-effects were observed, and the local control rate was encouraging. Furthermore, we discover that patients with $\mathrm{B} 90 \geq 65 \%$ and $\mathrm{B} 80 \geq 90 \%$ could achieve a higher local control rate. Mechanisms of P-SABR to induce high local control may include a bystander effect. ${ }^{26-28}$ Interestingly, even though the CI index is similar, the HI index of P-SABR is significantly higher, indicating that P-SABR plans are less homogeneous.

\section{Conclusion}

Our retrospective study suggests that P-SBRT, which can increase dose and BED with equivalent toxicity, could achieve encouraging local control rates with well-tolerated side-effects. B90 might be an indicator for higher local control rate. We are looking forward to more RCTs to prove the feasibility and to broaden the indications of P-SABR.

\section{Data sharing statement}

The datasets analyzed during the current study are available from the corresponding author on reasonable request.

\section{Disclosure}

The authors report no conflicts of interest in this work.

\section{References}

1. Siegel RL, Miller KD, Jemal A. Cancer statistics, 2017. CA Cancer J Clin. 2017;67(1):7-30.

2. Dubben HH, Thames HD, Beck-Bornholdt HP. Tumor volume: a basic and specific response predictor in radiotherapy. Radiother Oncol. 1998;47(2):167-174.

3. Werner-Wasik M, Swann RS, Bradley J, et al. Increasing tumor volume is predictive of poor overall and progression-free survival: secondary analysis of the Radiation Therapy Oncology Group 93-11 phase I-II radiation dose-escalation study in patients with inoperable non-small-cell lung cancer. Int J Radiat Oncol Biol Phys. 2008;70(2):385-390.

4. Timmerman R, McGarry R, Yiannoutsos C, et al. Excessive toxicity when treating central tumors in a phase II study of stereotactic body radiation therapy for medically inoperable early-stage lung cancer. J Clin Oncol. 2006;24(30):4833-4839.

5. Sampath S. Treatment: radiation therapy. Cancer Treat Res. 2016;170: 105-118. 
6. Timmerman RD, Paulus R, Galvin J, et al. Toxicity analysis of RTOG 0236 using stereotactic body radiation therapy to treat medically inoperable early stage lung cancer patients. Int J Radiat Oncol Biol Phys. 2007;69(3):S86.

7. Dunlap NE, Cai J, Biedermann GB, et al. Chest wall volume receiving $>30$ Gy predicts risk of severe pain and/or rib fracture after lung stereotactic body radiotherapy. Int J Radiat Oncol Biol Phys. 2010; 76(3):796-801.

8. Stephans K. Stereotactic body radiotherapy for stage I non-small cell lung cancer. Cleve Clin J Med. 2012;79(Electronic Suppl 1): eS26-eS31.

9. Feuvret L, Noel G, Mazeron JJ, Bey P. Conformity index: a review. Int J Radiat Oncol Biol Phys. 2006;64(2):333-342.

10. Albain KS, Swann RS, Rusch VW, et al. Radiotherapy plus chemotherapy with or without surgical resection for stage III non-small-cell lung cancer: a phase III randomised controlled trial. Lancet. 2009; 374(9687):379-386.

11. Curran WJ Jr, Paulus R, Langer CJ, et al. Sequential vs. concurrent chemoradiation for stage III non-small cell lung cancer: randomized phase III trial RTOG 9410. J Natl Cancer Inst. 2011;103(19):1452-1460.

12. Le Chevalier T, Arriagada R, Quoix E, et al. Radiotherapy alone versus combined chemotherapy and radiotherapy in unresectable non-small cell lung carcinoma. Lung Cancer. 1994;10(Suppl 1):S239-S244.

13. Nguyen NP, Bishop M, Borok TJ, et al. Pattern of failure following chemoradiation for locally advanced non-small cell lung cancer: potential role for stereotactic body radiotherapy. Anticancer Res. 2010 30(3):953-961.

14. Song CW, Kim MS, Cho LC, Dusenbery K, Sperduto PW. Radiobiological basis of SBRT and SRS. Int J Clin Oncol. 2014;19(4):570-578.

15. Nahum AE. The radiobiology of hypofractionation. Clin Oncol. 2015;27(5):260-269.

16. Reynders K, Illidge T, Siva S, Chang JY, De Ruysscher D. The abscopal effect of local radiotherapy: using immunotherapy to make a rare event clinically relevant. Cancer Treat Rev. 2015;41(6):503-510.

17. Sharabi AB, Lim M, DeWeese TL, Drake CG. Radiation and checkpoint blockade immunotherapy: radiosensitisation and potential mechanisms of synergy. Lancet Oncol. 2015;16(13):e498-e509.
18. Bernstein MB, Krishnan S, Hodge JW, Chang JY. Immunotherapy and stereotactic ablative radiotherapy (ISABR): a curative approach? Nat Rev Clin Oncol. 2016;13(8):516-524.

19. Barker HE, Paget JT, Khan AA, Harrington KJ. The tumour microenvironment after radiotherapy: mechanisms of resistance and recurrence. Nat Rev Cancer. 2015;15(7):409-425.

20. Song CW, Lee YJ, Griffin RJ, et al. Indirect tumor cell death after high-dose hypofractionated irradiation: implications for stereotactic body radiation therapy and stereotactic radiation surgery. Int J Radiat Oncol Biol Phys. 2015;93(1):166-172.

21. Chang JY, Senan S, Paul MA, et al. Stereotactic ablative radiotherapy versus lobectomy for operable stage I non-small-cell lung cancer: a pooled analysis of two randomised trials. Lancet Oncol. 2015;16(6): 630-637.

22. Moghanaki D, Chang JY. Is surgery still the optimal treatment for stage I non-small cell lung cancer? Transl Lung Cancer Res. 2016;5(2): 183-189.

23. Salazar OM, Sandhu TS, Lattin PB, et al. Once-weekly, high-dose stereotactic body radiotherapy for lung cancer: 6-year analysis of 60 early-stage, 42 locally advanced, and 7 metastatic lung cancers. Int J Radiat Oncol Biol Phys. 2008;72(3):707-715.

24. Feddock J, Arnold SM, Shelton BJ, et al. Stereotactic body radiation therapy can be used safely to boost residual disease in locally advanced non-small cell lung cancer: a prospective study. Int J Radiat Oncol Biol Phys. 2013;85(5):1325-1331.

25. Karam SD, Horne ZD, Hong RL, McRae D, Duhamel D, Nasr NM. Dose escalation with stereotactic body radiation therapy boost for locally advanced non small cell lung cancer. Radiat Oncol. 2013;8:179.

26. Asur R, Butterworth KT, Penagaricano JA, Prise KM, Griffin RJ. High dose bystander effects in spatially fractionated radiation therapy. Cancer Lett. 2015;356(1):52-57.

27. Klammer H, Mladenov E, Li F, Iliakis G. Bystander effects as manifestation of intercellular communication of DNA damage and of the cellular oxidative status. Cancer Lett. 2015;356(1):58-71.

28. Morgan WF, Sowa MB. Non-targeted effects induced by ionizing radiation: mechanisms and potential impact on radiation induced health effects. Cancer Lett. 2015;356(1):17-21.
OncoTargets and Therapy

\section{Publish your work in this journal}

OncoTargets and Therapy is an international, peer-reviewed, open access journal focusing on the pathological basis of all cancers, potential targets for therapy and treatment protocols employed to improve the management of cancer patients. The journal also focuses on the impact of management programs and new therapeutic agents and protocols on

\section{Dovepress}

patient perspectives such as quality of life, adherence and satisfaction. The manuscript management system is completely online and includes a very quick and fair peer-review system, which is all easy to use. Visit http://www.dovepress.com/testimonials.php to read real quotes from published authors. 